\title{
Complete Response by Computed Tomography
}

National Cancer Institute

\section{Source}

National Cancer Institute. Complete Response by Computed Tomography. NCI

Thesaurus. Code C123577.

The disappearance of all signs of cancer, based on CT scan and criteria-defined assessments. 